\title{
Facile way for synthesis silver nanoparticles for obtaining antibacterial textile fabrics
}

\author{
Ahmed Saad Montaser*, Mohamed A Ramadan, Ali Ali Hebeish \\ Textile Research division, Preparation and finishing, National research Centre, Dokki, Giza, Egypt.
}

\section{ARTICLE INFO \\ Article history: \\ Received on: 27/12/2015 \\ Revised on: 27/02/2016 \\ Accepted on: 14/04/2016 \\ Available online: 28/06/2016}

Key words:

PVA, $\mathrm{NaBH}_{4}$, AgNPs,

Antibacterial, Textiles.

\begin{abstract}
One pot synthesis of silver nanoparticles (AgNPs) with well-defined size using polyvinyl alcohol (PVA) as stabilizing agent and sodium borohydride $\left(\mathrm{NaBH}_{4}\right)$ as reducing agent was successfully performed. Preparation of AgNPs was carried out under a variety of conditions including concentrations of AgNPs, PVA and $\mathrm{NaBH}_{4}$. UV-vis spectra and TEM images were employed to characterize AgNPs obtained under the said different conditions. Physical and mechanical properties of cotton/polyester fabric treated with the synthesized silver nanoparticles were measured. SEM and EDX were used to scanning and observe the morphology and elemental change pertaining to fabric surface. Antibacterial activity against E. coli ( $\mathrm{Gm}$-ve bacteria) and Staphylococcus aureus $(\mathrm{Gm}+\mathrm{ve}$ bacteria) were successfully examined against the treated and untreated fabrics.
\end{abstract}

\section{INTRODUCTION}

The preparation of metal nanoparticles is a major research area in nanoscale science and engineering (Hebeish et al., 2013). This is rather emphasized by virtue of the unusual chemical and physical properties of the metal nanoparticles vis á - vis these properties when the metal is in the bulk state. Among the unique properties of the metal nanoparticles are catalytic activity, novel electronic, optic and magnetic properties and their potential application in biotechnology. Numerous studies have been reported on the preparation of new organic/inorganic hybrid materials and nanocomposites such as metal-polymer nanoparticles(Elghanian et al., 1997). AgNPs have also attracted much attention due to their diminutive size and novel material properties. With their nanometer scale size, which is responsible for different properties when compared with

\section{* Corresponding Author}

A. S. Montaser, Preparation and finishing Department, Textile Research Division, National Research Center. Dokki, Giza, Egypt.

Email:dr_asaadnrc@yahoo.com, Phone No: +201144409443 the bulk material, renders them suitable for applications(Ray, 2010). Indeed, many approaches have been undertaken to prepare AgNPs for a rapidly growing list of catalysis, electronic, non-linear optics and biomaterial applications (Huang and Yang, 2004; Je et al., 2006; Sugimoto et al., 1998; Twu et al., 2008; Wei and Qian, 2008). In the preparation of AgNPs, the stabilizer plays an important role in retaining the stabilization of Nanosystem.

The antimicrobial properties of silver ions have been known since ancient times and silver ions are widely used as bactericide in surgical prostheses and dental implants (El-Rafie et al., 2010; Hebeish et al., 2011; Hebeish et al., 2014; Sulaiman et al., 2013; Wei et al., 2009). Many methods have been used for the preparation of nanoparticles including microbial, chemical and physical methods. The most popular among them is the chemical reduction of silver salts by sodium citrate. AgNPs show optical properties, which are not observed neither in molecules nor in bulk metals (Rivas et al., 2001). The formation of the AgNPs can be monitored using UV-vis absorption spectroscopy since it exhibits the typical surface plasmon absorption maxima at $418-420 \mathrm{~nm}$ from the UV-vis spectrum (Guzman et al., 2012). 
PVA was used in various applications in textile industry; however, to the author's knowledge no systematic study for preparation of the AgNPs using PVA has been published so far. It is the essential aim of current work to present detailed and integrated investigations pertaining to the preparation of AgNPs with well-defined size using PVA as stabilizing agent for silver ions after reducing by $\mathrm{NaBH} 4$ by insitu reaction. Preparation of AgNPs was carried out under a variety of conditions including concentrations of AgNPs and PVA. UV-vis spectra, TEM images are employed to characterize AgNPs obtained under the said different conditions. Mechanical properties of cotton/ polyester fabric treated with the prepared silver nanoparticles were measured. The antibacterial activity of the prepared fabrics loaded AgNPs was also measured against the two types of bacteria.

\section{MATERIALS AND METHODS}

\section{Preparation of silver nanoparticles (AgNPs) using polyvinyl} alcohol (PVA) and sodium borohybride $\left(\mathrm{NaBH}_{4}\right)$.

PVA with different concentration (0-1gm /100 ml) was dissolved in distilled water using heating magnetic stirrer. After complete dissolution, the $\mathrm{pH}$ of the solution was adjusted to $\mathrm{pH}$ 7.Also $0.1 \mathrm{~N}\left(\mathrm{NaBH}_{4}\right)$ was added with different concentrations (25$175 \mu \mathrm{l})$ to the reaction medium followed by drop-wise addition of a different amount of $0.1 \mathrm{~N}$ AgNPs solution (50-350 $\mu \mathrm{l}$ ) (keeping in mind that the total volume of the reaction medium is $100 \mathrm{ml}$ ). The reaction mixture was kept under continuous stirring for (15 min).The reaction medium acquires a clear dark green color indicating the formation of silver nanoparticles. The progression of the reaction was controlled by UV-vis absorption; aliquots from the reaction bulk were withdrawn at given time intervals and evaluated.

\section{Polyester/ cotton fabric treatment with obtained AgNPs.}

Polyester/cotton fabrics treated using pad-dry cure method. Thus fabrics were padded with the prepared AgNPs in aqueous solutions (50 and 3500) PPm with a wet pick up of $100 \%$. The treated fabrics were dried at $100{ }^{0} \mathrm{C}$ for $30 \mathrm{~min}$ followed by thermofixation at $150{ }^{\circ} \mathrm{C}$ for $3 \mathrm{~min}$. The cured fabric samples were thoroughly washed with cold water, neutralized and dried in air (Abdel-Mohsen et al., 2012).

\section{Testing and Analysis}

The characterization of the prepared product in each step and the performance properties of the treated fabrics were evaluated using the following published standard procedures:

\section{Ultra Violet-Visible (UV-Vis) Spectra}

UV-vis spectral analysis was done by using a 50 ANALYTIKA, JENA Spectrophotometer from 200 to $500 \mathrm{~nm}$.

\section{Transmission Electron Microscope (TEM)}

TEM was used to assess the potential impact of the modification on the elemental and structural properties of the synthesized AgNPs nanoparticles, whether alone or over and within the fabric surface. The TEM analysis was done using JEOL-JEM-1200 (Japan).

\section{Scanning electron microscope and energy dispersive x-ray analysis (SEM and EDX)}

SEM analysis was done using a scanning electron probe micro analyzer (JXA-840A, Japan). The specimens in the form of films were mounted on the specimen stabs and coated with thin film of gold by the sputtering method. The micrograph was taken at magnification of 1000 using (KV) accelerating voltage. The composition of selected nanoparticles was determined by energy dispersive X-ray (EDX) analysis using INCa (X Sight) (Azeem et al., 2015).

\section{Performance and Testing of the Cotton Fabrics Color strength (K/S) and fastness}

Color strength expressed as $(\mathrm{K} / \mathrm{S})$, and the fastness properties, washing, perspiration and rubbing, were assessed according to standard methods.

\section{Tensile Properties}

Polyester/cotton fabrics were tested for the change in their tensile properties before and after treatment. The breaking load of the samples were determined by the strip method on tensile tester instrument (ASANO Machine MFG.CO, DSAKA, No.6202, Japan ). According to ASTM method D2256-66T (ASTM Test Method, 1972). Elongation- at-break was determined according to ASTM procedure D-2296-66T (Abdel-Mohsen et al., 2012).

\section{Antimicrobial Activity Test}

Test disc diffusion method with some modification was used for screening test of the cotton fabric sample for antimicrobial activity. Nutrient agar for bacteria $0.1 \mathrm{ml}$ of an appropriate dilution of the test culture was used. The treated fabric samples $\left(\mathrm{cm}^{-1}\right.$ diameter $)$ were placed on the surface of the incubated plates at $35^{\circ} \mathrm{C}$ for 24 hours. Diameter of plates inhibition zone $(\mathrm{mm})$ including the disc diameter was measured for each treatment (Abdel-Mohsen et al., 2011).

\section{RESULTS AND DISCUSSION}

\section{Silver nanoparticles Characterization}

The reduction of silver ions in aqueous solutions generally yields colloidal silver with particles diameter of several nanometers. These particles have specific optical properties indicated by the presence of intense absorption band around 400 $\mathrm{nm}$ caused by collective excitation of all the free electrons in the particles. Metal atoms formed by reduction tend to coalesce into oligomers which themselves progressively grow into larger clusters. However, the coalesce must be limited by adding a polymeric molecule acting as cluster stabilizer. Functional groups with high affinity to metal ensure the anchoring of the molecule at the cluster surface while the polymeric chain protects cluster from 
coalescing with the next one through electrostatic repulsion or steric hindrance (Abdel-Mohsen et al., 2012). PVA was selected as a capping agent as it only stabilizes but does not reduce ions. The reducing agent $\mathrm{NaBH}_{4}$ has ability to donate electrons when dissolving in water helping the metal ions to complete its outer valance shell to convert to metal nanoparticles.

\section{Effect of presence and absence of PVA of AgNPs preparation}

Fig. 1 shows the UV- vis spectra of the silver colloid obtained using $\mathrm{NaBH}_{4}$ as reducing agent and with presence and absence of PVA which functions as stabilizing agent. The spectra reveal a number of observations which may be summarized as follows: a) in absence of PVA the reduction process can't be completed correctly and presence of aggregation can be observed from the broad band of silver nanoparticles at 397 $\mathrm{nm}$; there's presence of silver salt appeared at the end of the spectra. b) In presence of PVA the reduction process provided completely and sharp band already appeared at $400 \mathrm{~nm}$. These observations stated above could be attributed to the reduction process of silver ions to silver atom using $\mathrm{NaBH}_{4}$ followed by anchoring between AgNPs at the cluster surface, while the polymeric chain protects the cluster from fusion with the next silver molecule.

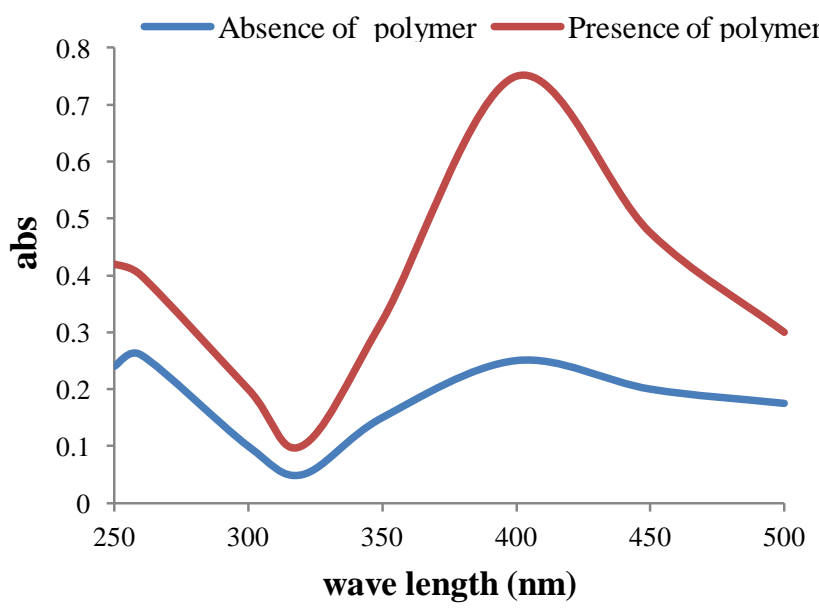

Fig. 1: UV- vis spectra of the AgNPs where in absence and presence of PVA.

\section{Effect of PVA concentration on AgNPs preparation}

Fig. 2 shows the UV-vis absorption spectra of silver nanoparticles prepared using PVA at different concentrations (0$1 \mathrm{gm} / 100 \mathrm{ml}$ ). The data reveal that regardless of the PVA concentration used, similar Plasmon bands are formed at wavelength $408 \mathrm{~nm}$ with the formation of the ideal bell shape which is characteristic for the formation of $\mathrm{Ag}^{0}$ nanoparticles. It is clear also that there is a gradual increase in the absorption intensity, by increasing the PVA concentration up to $0.75 \mathrm{gm}$ $/ 100 \mathrm{ml}$ which could be ascribed to the enhancement in the stabilization efficiency of the formed silver nanoparticles. Over this concentration the reduction efficiency decreased because presence of PVA is created. In this case, the UV - vis spectra may represent the highly viscose solution of PVA that would act as a barrier between silver salt molecules and the $\mathrm{NaBH}_{4}$ thereby decreasing the AgNPs formation.

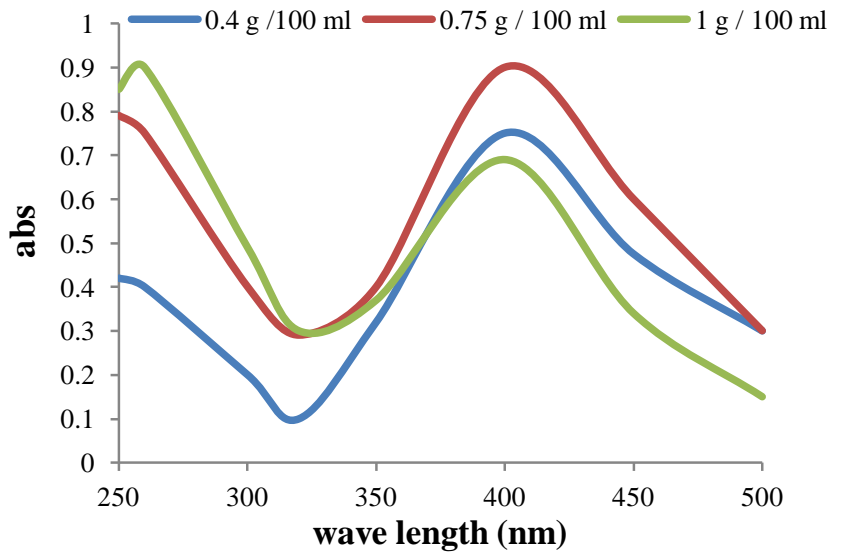

Fig. 2: UV-Vis absorption spectra of AgNPs at different concentrations of PVA.

\section{Effect of $\mathrm{AgNO}_{3}: \mathrm{NaBH}_{4}$ ratio on silver nanoparticles preparation}

In order to achieve better stability and efficient reduction for conversion of silver ions to silver nanoparticles with extremely small sizes, certain ratio of AgNPs to $\mathrm{NaBH}_{4}$ in the reaction medium must be established at the optimum condition of PVA concentration. Fig.3a showing that absorbance intensity of the prepared AgNPs increases with increasing amount of $\mathrm{AgNO}_{3}$ against $\mathrm{NaBH}_{4}$ upto $50 \mathrm{PPm}$ then tends to decreases. Hence, preparation of silver nanoparticles was carried out using higher concentrations of both $\mathrm{AgNO}_{3}$ and reducing agent. Increasing the concentrations of both $\mathrm{AgNO}_{3}$ and $\mathrm{NaBH}_{4}$ was done while keeping the ratio of the two component constant.

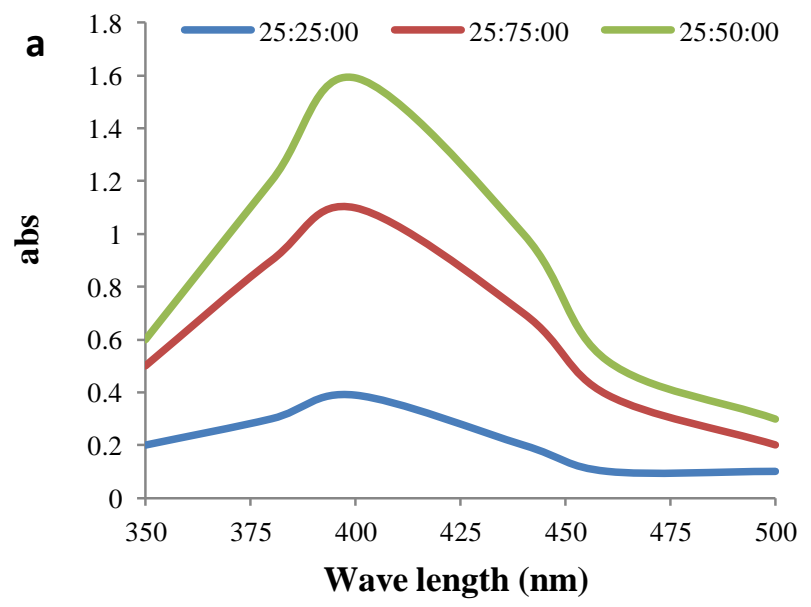

Fig. $3 b$ shows the UV-vis spectra of AgNPs resulting from incorporating different concentrations of AgNPs and $\mathrm{NaBH}_{4}$ while keeping the ratio between them always constant. UV spectra reveals that similar absorption spectra are obtained at wavelength $408 \mathrm{~nm}$ with marginal increase in the intensity of the absorption peak by increasing amounts of $\mathrm{AgNO}_{3}$ and $\mathrm{NaBH}_{4}$ incorporated in the reaction medium. In addition to that, the UV-vis spectra 
showed nearly the disappearance of the peak characteristic for silver ion, until reaching $350 \mu \mathrm{l} 0.1 \mathrm{~N} \mathrm{AgNO}_{3}$ and $175 \mu \mathrm{l} 0.1 \mathrm{~N}$ $\mathrm{NaBH}_{4}$ the Plasmon of silver peak tend to decrease and may be related to complete reduction of the silver salt.

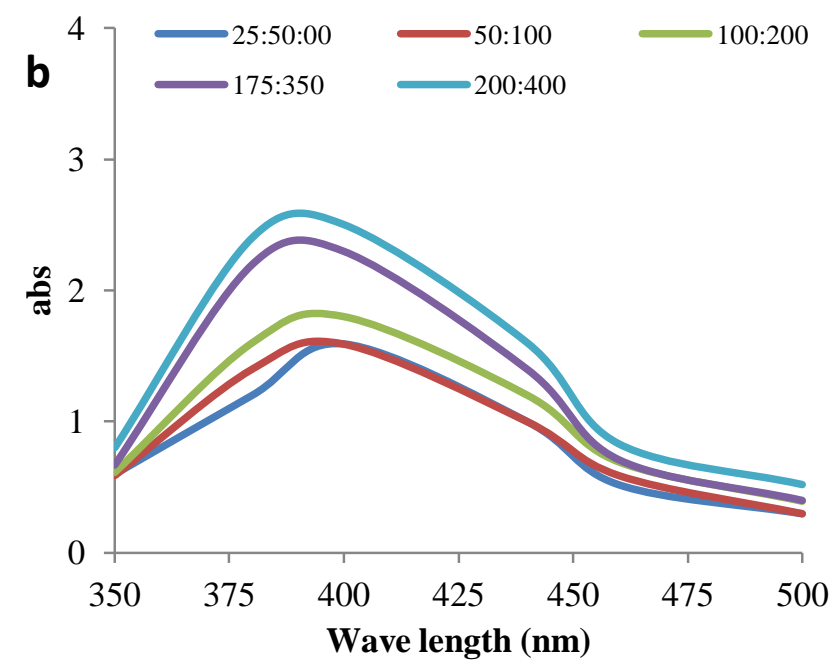

Fig. 3: UV-vis spectra of AgNPs obtained to a) different concentrations $\mathrm{AgNO}_{3}$ and $\mathrm{NaBH}_{4}$ with constant ratio. b) Ascending ratios of $\mathrm{AgNO}_{3}: \mathrm{NaBH}_{4}$.

\section{TEM images of the concentrated and diluted AgNPs}

Fig.4 shows transmission electron microscope images of concentrated and diluted silver nanoparticles (3500 and $50 \mathrm{PPm}$ ). The data reveal that regardless of the AgNPs concentration, the particle size distributions lies in nanosized range from $10-100 \mathrm{~nm}$ are found in TEM images.
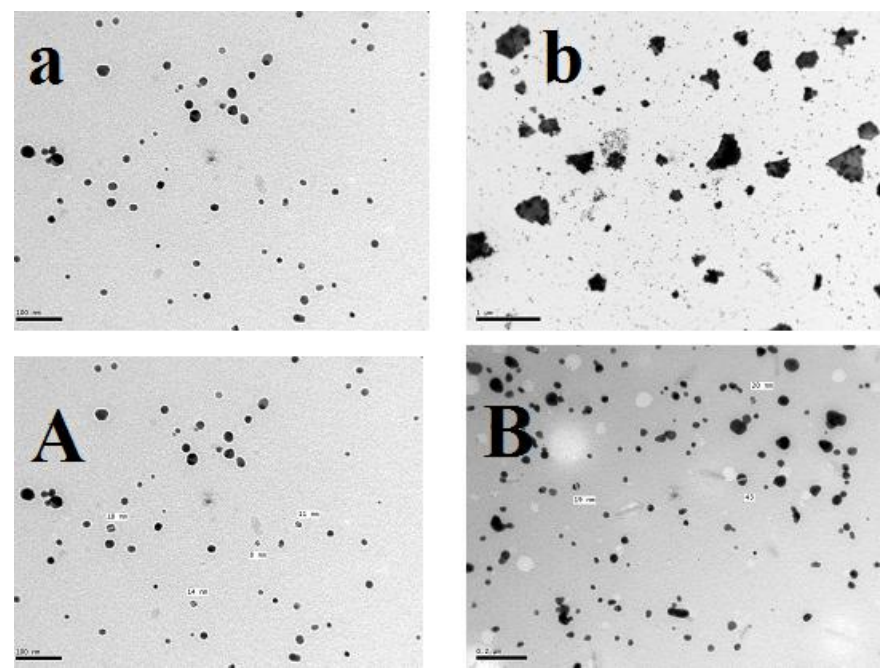

Fig. 4: TEM images of concentrated and diluted AgNPs where, a) 50 and b) $3500 \mathrm{ppm}$.

The particles size increases with increasing the amount of $\mathrm{AgNO}_{3}$ which used in AgNPs preparation. It is understandable that the increase in AgNPs concentration accompanied by an increase of the PVA concentration. This could be ascribed to the enhancement in the stabilization efficiency of the formed silver nanoparticles until a definite range is attained, after which the effect of PVA is devoted to agglomeration of the prepared silver nanoparticles.

\section{SEM and EDX}

The surfaces of polyester/ cotton fabrics were morphologically observed by SEM. Fig.5 shows the surface appearances of silver nanoparticles treated sample and the untreated one. The photographs reveal that before the treatment (Fig 5a) polyester/ cotton fiber surface was smooth and flat, while the finished fiber surface (Fig. 5b) has an appearance of small grains as well as the fiber mesh layer. From the SEM pictures, it is clear that the treatment of polyester/ cotton with PVA /AgNPs composite results in a unique morphological form, having a more textured surface than the unmodified one. This result is caused by the deposition of PVA on the surface of polyester/ cotton fabric by crosslinking agent.

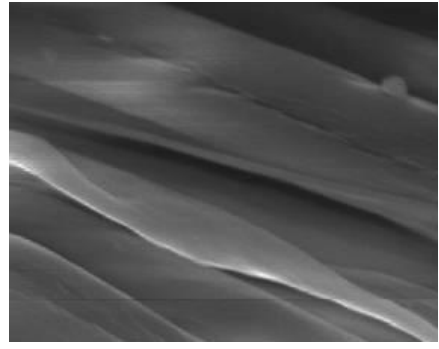

A

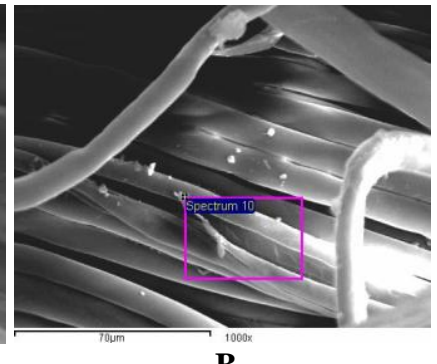

B
Fig. 5: SEM of the a) untreated fabric and b) treated fabric with AgNPs.

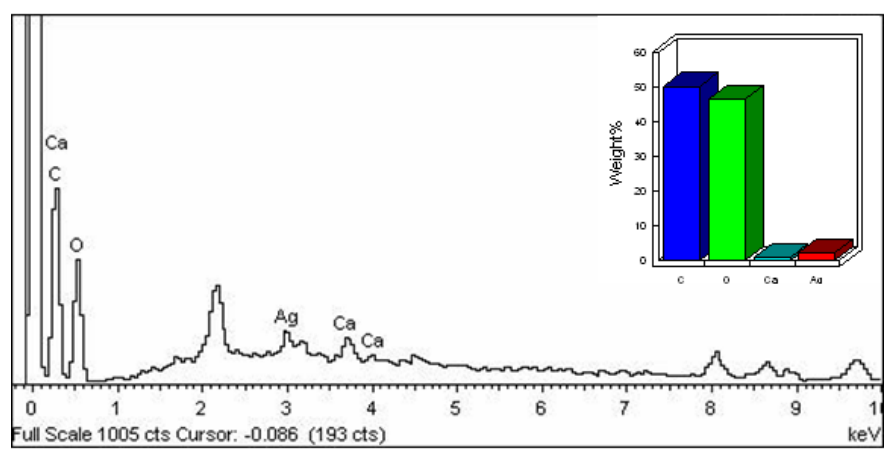

Fig 6: EDX of the treated fabrics with AgNPs.

The incorporation of silver nanoparticles in the polyester/ cotton fabrics was also verified by SEM-EDX Fig. 6where the polyester/ cotton fibers containing silver nanoparticles are observed in association with the presence of the silver metal peak.

\section{Color measurement}

The yellowness indexes of the untreated and treated polyester/cotton fabrics with different concentrations of AgNPs are presented in figure 7. The yellowness indexes are measured primarily to study the yellowing effect of AgNPs treatment concentrations. The yellowness index measurements show that loading of $\mathrm{Ag}$ nanoparticles on polyester/cotton fabrics lead to increase in yellowness. Higher AgNPs concentration led to higher yellowness indexes (Haji et al., 2013). The presence of low 
concentration of AgNPs resulted in increasing of the fabric whiteness. Therefore, the higher concentration of AgNPs applied on the loaded polyester/cotton fabrics led to yellowness appearance and vis-à-vis.

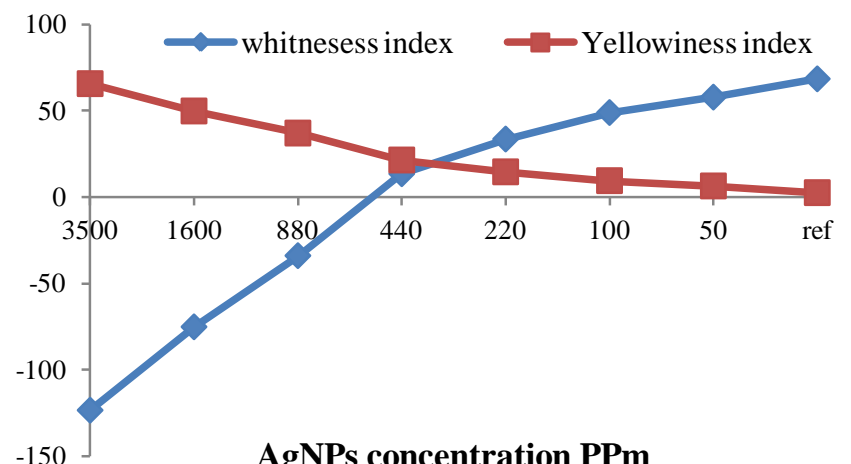

Fig. 7: Yellowness and Whiteness index of the untreated and treated cotton fabric with different concentrations of AgNPs.

\section{Mechanical properties}

Textile mechanical properties of the treated viscose/ polyester fabrics display significant improvement when measured by crease recovery of the fabrics after treatment in presence of different concentrations of AgNPs. Figure 8 a, b shows fabrics tensile strength and elongation at break properties are increase after treatment.
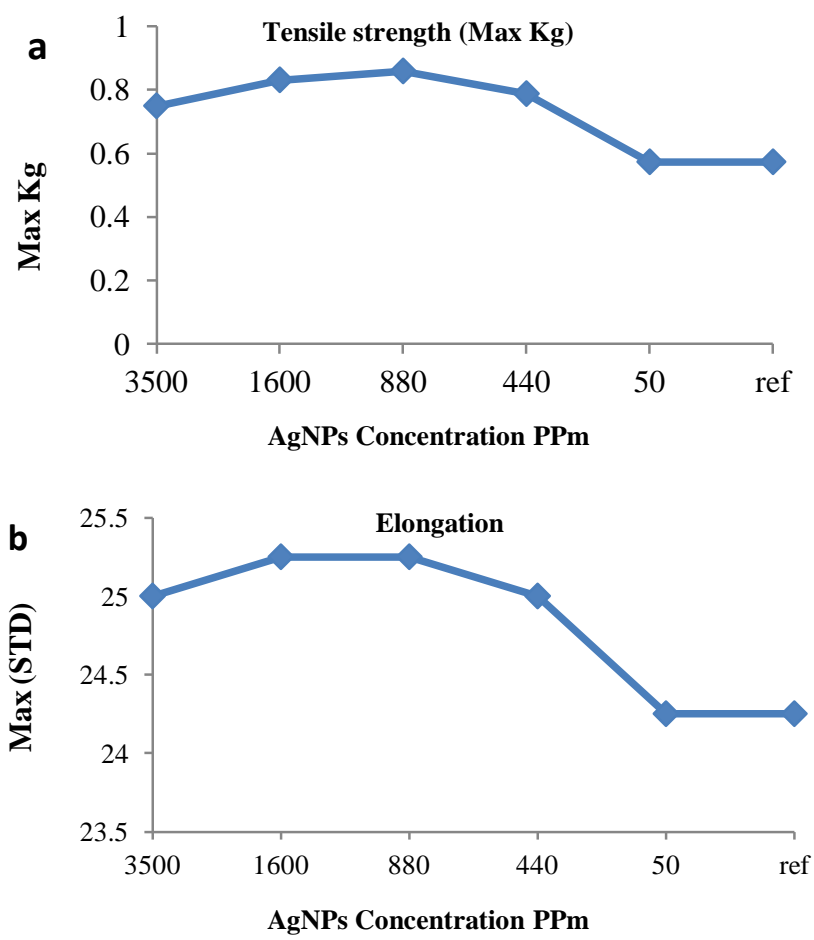

Fig. 8: Mechanical strength of treated and untreated fabrics with different concentrations of AgNPs. Where a) Tensile strength, b) Elongation at break.

It can be explained by the nanoparticles which coated the fibers and penetrate them, thus making the fabrics more durable and long lasting. The properties increase with decreasing AgNPs concentration until $880 \mathrm{PPm}$. This can be attributed to the decrease spaces between the fibers after treating with high concentrations, of AgNPs resulting in decrease extensibility. Therefore the properties increases with dilution until reach 880 PPm. Further dilution of the AgNPs decreases the tensile properties.

\section{Antibacterial activity}

The antibacterial activity of AgNPs /PVA was studied against E. coli and Staph. aureus as shown in the Fig.9. The growth of $\mathrm{E}$. coli and $\mathrm{S}$. aureus was affected negatively by AgNPs/PVA treated fabric compared with the untreated one. The inhibition zone could be seen clearly around the treated fabrics, it's around $16 \mathrm{~mm}$ in the case of $E$. coli and $15 \mathrm{~mm}$ in the case of Staph. aureus. The behavior can be attributed to the presence of silver nanoparticles and their antibacterial activity.

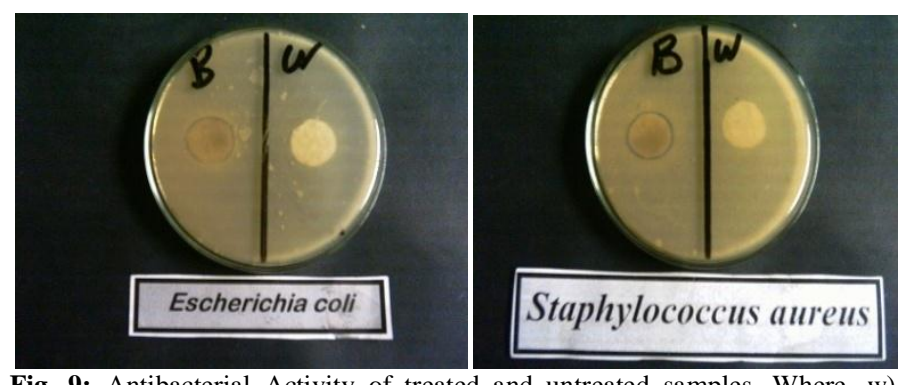

Fig. 9: Antibacterial Activity of treated and untreated samples. Where, w) untreated fabrics and B) treated fabrics.

\section{CONCLUSION}

Silver nanoparticles could successfully be prepared through a simple and facile in situ regeneration method. The formation of silver nanoparticles was assessed by UV- vis and TEM. The morphological change identified using SEM and EDX. The optimum condition for obtaining homogenous and concentrated AgNPs as follows: 0.75 gm PVA/100 ml; $350 \mu \mathrm{l} 0.1$ $\mathrm{N} \mathrm{AgNO}_{3}$ and $175 \mu \mathrm{l} 0.1 \mathrm{~N} \mathrm{NaBH}_{4}$. It is shown to have an efficient bactericidal effect after AgNPs were applied to textile fabrics. The positive reflection of AgNPs concentrations on treating process of the polyester/cotton fabric on mechanical properties was also reported. The major advantages were feasibility of the synthesis of the silver nanoparticles as the one pot synthesis serves time and power use, a point which makes it attractive, especially for textile applications.

\section{REFERENCES}

Abdel-Mohsen AM, Aly AS, Hrdina R, Montaser AS, Hebeish A. Eco-Synthesis of PVA/Chitosan Hydrogels for Biomedical Application. Journal of Polymers and the Environment, 2011; 19: 10051012.

Abdel-Mohsen AM, Aly AS, Hrdina R, Montaser AS, Hebeish A. Biomedical Textiles Through Multifunctioalization of Cotton Fabrics Using Innovative Methoxypolyethylene Glycol-N-Chitosan Graft Copolymer. Journal of Polymers and the Environment, 2012; 20: 104-116.

Abdel-Mohsen AM, Abdel-Rahman RM, Hrdina R, Imramovský A, Burgert L, Aly AS. Antibacterial cotton fabrics treated 
with core-shell nanoparticles. International Journal of Biological Macromolecules, 2012; 50: 1245-1253.

Abdel-Mohsen AM, Hrdina R, Burgert L, Krylová G, AbdelRahman RM, Krejčová A, Steinhart M,Beneš L. Green synthesis of hyaluronan fibers with silver nanoparticles. Carbohydrate Polymers, 2012; 89: 411-422.

Azeem RaE, Nada AA, Montaser AS. Chitosan Liposomal microspheres for Ricinoleic acid Encapsulation. J App Pharm Sci, 2015; 5: 055-062.

El-Rafie MH, Mohamed AA, Shaheen T, Hebeish A. Antimicrobial effect of silver nanoparticles produced by fungal process on cotton fabrics. Carbohydrate Polymers, 2010; 80: 779-782.

Elghanian R, Storhoff, Mucic RC, Letsinger RL, Mirkin CA. Selective Colorimetric Detection of Polynucleotides Based on the Distance-Dependent Optical Properties of Gold Nanoparticles. Science, 1997; 277: 1078-1081.

Guzman M, Dille J, Godet S. Synthesis and antibacterial activity of silver nanoparticles against gram-positive and gram-negative bacteria. Nanomedicine: Nanotechnology, Biology and Medicine, 2012; 8: $37-45$.

Haji A, Qavamnia S, Barani H. In situ synthesis and loading of silver nanoparticles on cotton fabric. Industria Textila, 2013; 64: 8-12.

Hebeish A, El-Naggar ME, Fouda MMG, Ramadan MA, AlDeyab SS,El-Rafie MH. Highly effective antibacterial textiles containing green synthesized silver nanoparticles. Carbohydrate Polymers, 2011; 86: 936-940.

Hebeish AA, Ramadan MA, Montaser AS, Farag AM. Preparation, characterization and antibacterial activity of chitosan-g-poly acrylonitrile/silver nanocomposite. International Journal of Biological Macromolecules, 2014; 68: 178-184.

Hebeish AA, Aly AS, Ramadan MA, Abd El-Hady MM, Montaser AS, Farag AM. Establishment of optimum conditions for preparation of silver nanoparticles using carboxymethyl chitosan. Egyptian Journal of Chemistry, 2013; 56: 241-254.

Huang H,Yang X. Synthesis of polysaccharide-stabilized gold and silver nanoparticles: a green method. Carbohydrate Research, 2004; 339: $2627-2631$
Je J-Y, Cho Y-S, Kim S-K. Cytotoxic activities of water-soluble chitosan derivatives with different degree of deacetylation. Bioorganic \& Medicinal Chemistry Letters, 2006; 16: 2122-2126.

Ray PC. Size and Shape Dependent Second Order Nonlinear Optical Properties of Nanomaterials and Their Application in Biological and Chemical Sensing. Chemical Reviews, 2010; 110: 5332-5365.

Rivas L, Sanchez-Cortes S, García-Ramos JV, Morcillo G. Growth of Silver Colloidal Particles Obtained by Citrate Reduction To Increase the Raman Enhancement Factor. Langmuir, 2001; 17: 574-577.

Sugimoto M, Morimoto M, Sashiwa H, Saimoto H,Shigemasa Y. Preparation and characterization of water-soluble chitin and chitosan derivatives. Carbohydrate Polymers, 1998; 36: 49-59.

Sulaiman GM, Mohammed WH, Marzoog TR, Al-Amiery AA, Kadhum AH, Mohamad AB. Green synthesis, antimicrobial and cytotoxic effects of silver nanoparticles using Eucalyptus chapmaniana leaves extract. Asian Pacific Journal of Tropical Biomedicine, 2013; 3: 58-63.

Twu Y-K, Chen Y-W,Shih C-M. Preparation of silver nanoparticles using chitosan suspensions. Powder Technology, 2008; 185: 251-257.

Wei D, Qian W. Facile synthesis of Ag and Au nanoparticles utilizing chitosan as a mediator agent. Colloids and Surfaces B: Biointerfaces, 2008; 62: 136-142.

Wei D, Sun W, Qian W, Ye Y, Ma X. The synthesis of chitosan-based silver nanoparticles and their antibacterial activity. Carbohydrate Research, 2009; 344: 2375-2382.

\section{How to cite this article:}

Montaser AS, Ramadan MA, Hebeish AA. Facile way for synthesis silver nanoparticles for obtaining antibacterial textile fabrics. J App Pharm Sci, 2016; 6 (06): 139-144. 